\title{
Optical sectioning by two-pinhole confocal fluorescence microscopy
}

\author{
M. Martínez-Corral ${ }^{\mathrm{a}, *}$, M.T. Caballero ${ }^{\mathrm{b}}$, C. Ibáñez-López ${ }^{\mathrm{a}}$, V. Sarafis ${ }^{\mathrm{c}}$ \\ a Departamento de Óptica, Universidad de Valencia, Burjassot 46100, Spain \\ ${ }^{\mathrm{b}}$ Departamento de Óptica, Universidad de Alicante, Alicante 03080, Spain \\ ${ }^{\mathrm{c}}$ School of Computing and Electrical Imaging, University of Queensland, St Lucia, Qld 4072, Australia
}

\begin{abstract}
A two-pinhole axially superresolving confocal fluorescence imaging system is presented. Based on the concept of subtractive incoherent imaging, the system described here is equipped with a zero-focus complex-transmittance pupil filter in one of the collector paths. The optical sectioning capacity of the system is $25 \%$ superior to that of a free-pupil one-pinhole instrument.

(C) 2003 Elsevier Ltd. All rights reserved.

Keywords: Confocal microscopy; Fluorescence; Point-spread function
\end{abstract}

\section{Introduction}

The main feature of confocal scanning microscopy is its familiar optical sectioning capacity when imaging threedimensional (3D) fluorescence samples (Wilson, 1990). However, as it is well known, the axial resolution of such setups is much poorer than its lateral counterpart. Along the past few years several attempts have been done to improve the axial resolution by means of the so-called point-spread function (PSF) engineering method. In this context, we can cite the 4Pi confocal fluorescence technique (Hell and Stelzer, 1992) in which a substantial narrowing of the axial PSF main peak is obtained by the interference of two opposing tightly focused wave fronts. The use of purely absorbing (Martínez-Corral et al., 1999) or complextransmittance pupil filters (Neil et al., 2000; Sheppard, 1999) has also been proposed to reach the same end.

An alternative approach to improve the resolution capacity of confocal scanning imaging systems is the subtractive imaging technique. In this technique, a superresolving synthetic image is obtained by means of a twopinhole confocal setup (Boyer and Sarafis, 2001). However, as far as we know this technique has been applied only to improve the transverse resolution.

In this paper, we present an extension of the concept of two-pinhole confocal imaging to the $3 \mathrm{D}$ case. The proposed

\footnotetext{
* Corresponding author. Tel.: + 34-96-386-4343; fax: + 34-96-386-4715.

E-mail address: manuel.martinez@uv.es (M. Martínez-Corral).
}

setup consists of an illumination arm that focuses onto the sample the light proceeding from a monochromatic point source. The collected fluorescent light is split into two beams, which are independently focused onto two different pinholed detectors. A linear combination of the two acquired confocal images provides a synthetic image in which the optical sectioning is considerably enhanced as compared with the images provided by a conventional one-pinhole confocal setup. The cornerstone of our system is the insertion, in one of the collector paths, of a properly designed zero-focus complex-transmittance pupil filter. These filters belong to the class of destructive-interference apodizers (MartínezCorral et al., 1998). The design procedure of the filter takes into account both the numerical aperture of the objective and the ratio between the illumination and the fluorescence wavelengths. As we show below the proposed system permits a reduction in the axial extent of the integrated intensity function (as measured at $1 \%$ of its maximal value) to about $75 \%$ of that corresponding to a conventional confocal setup. This leads to a significant improvement of the optical sectioning. The utility of our method is illustrated by means of a numerical imaging experiment.

\section{Two-pinhole confocal setup}

Consider the confocal-scanning setup schematically plotted in Fig. 1. In the system, a fluorescent specimen is 


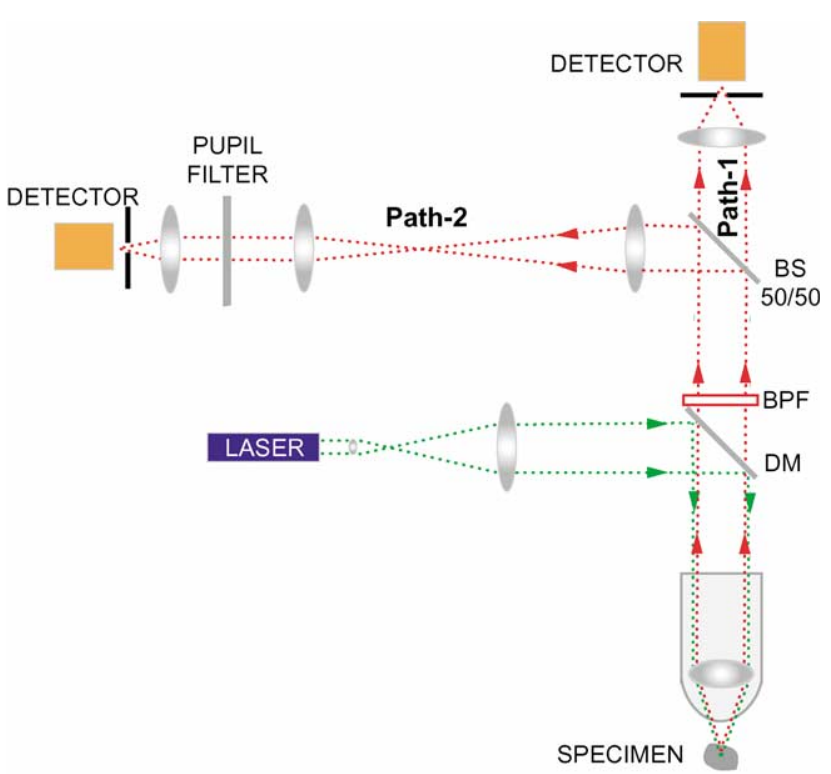

Fig. 1. Schematic lay out of the two-pinhole confocal arrangement. The specimen is illuminated by the light proceeding from a monochromatic point source. The fluorescent light is independently collected by two different pinholed detectors. Relay lenses in path 2 are used to allow the pupil filter to overlap exactly the exit pupil of the objective. BS: beam splitter, DM: dichroic mirror, BPF: bandpass filter.

illuminated by the tightly focused wave proceeding from a monochromatic point source. The fluorescence light emitted by the specimen is collected by the same objective. To avoid scattered laser light entering the detection path, a combination of a dichroic mirror and a bandpass filter is used. Then the fluorescent light is split into two beams, which are independently focused onto two different pinholed detectors. The relay lenses in path 2 have the appropriate magnification factor in order to overlap exactly the exit pupil of the objective. The system allows the parallel acquisition of two different images of the same object. The first image is obtained with a confocal configuration where both the illumination and the collector path are free pupil (path 1). In the second image the collector path is equipped with a pupil mask (path 2). The 3D intensity distribution corresponding to the resulting confocal images, can be expressed as

$$
\begin{aligned}
I_{1}(r, z) & =\left\{\left|h_{\mathrm{ill}}(r, z)\right|^{2}\left|h_{\mathrm{det} 1}(\varepsilon r, \varepsilon z)\right|^{2}\right\} \otimes_{3} O(r, z) \\
& =\operatorname{PSF}_{1}(r, z) \otimes_{3} O(r, z) \\
I_{2}(r, z) & =\left\{\left|h_{\mathrm{ill}}(r, z)\right|^{2}\left|h_{\mathrm{det} 2}(\varepsilon r, \varepsilon z)\right|^{2}\right\} \otimes_{3} O(r, z) \\
& =\operatorname{PSF}_{2}(r, z) \otimes_{3} O(r, z) .
\end{aligned}
$$

In the above equations $h_{\text {ill }}(r, z)$ stands for the amplitude PSF of the illumination path, and $h_{\text {det } i}(\varepsilon r, \varepsilon z)$ are the PSFs of paths 1 and 2 collection arms. Further, $O(r, z)$ is the $3 \mathrm{D}$ function that describes the spatial distribution of the fluorescence generation.
According to the scalar Debye theory, the amplitude PSF's are related to the pupil functions by

$$
h_{i}(r, z)=\int_{0}^{\alpha} A_{i}(\theta) J_{0}\left(\frac{2 \pi}{\lambda_{\mathrm{exc}}} n r \sin \theta\right) \exp \left(i 2 \pi n \frac{\cos \theta}{\lambda_{\mathrm{exc}}} z\right) \sin \theta \mathrm{d} \theta,
$$

where $0 \leq \theta \leq \alpha$ stands for the angular aperture, and $A_{i}(\theta)$ is the apodization function, i.e. the amplitude transmittance of the aperture stop. Function $A_{i}(\theta)$ is constant in the interval $\theta \in[0, \alpha]$ in illumination and collector path 1 . The parameter $\varepsilon=\lambda_{\text {exc }} / \lambda_{\text {det }}$ accounts for the fact that, due to the difference of energy between the fluorescence and the incident photons, the fluorescence wavelength, $\lambda_{\text {det }}$, is slightly larger than $\lambda_{\text {exc }}$.

The two-pinhole setup provides two independent parallely acquired images of the same specimen. Our proposal is to create a synthetic image by linearly combining the independent images. The synthetic image is

$I_{\text {syn }}(r, z)=I_{1}(r, z)-\gamma I_{2}(r, z)=\operatorname{PSF}_{\text {syn }}(r, z) \otimes_{3} O(r, z)$,

where

$$
\begin{aligned}
& \operatorname{PSF}_{\mathrm{syn}}(r, z)=\mathrm{PSF}_{1}(r, z)-\gamma \mathrm{PSF}_{2}(r, z)=\left|h_{\mathrm{ill}}(r, z)\right|^{2} \\
& \quad \times\left|h_{\mathrm{det} 1}(\varepsilon r, \varepsilon z)\right|^{2}-\gamma\left|h_{\mathrm{ill}}(r, z)\right|^{2}\left|h_{\mathrm{det} 2}(\varepsilon r, \varepsilon z)\right|^{2}
\end{aligned}
$$

where $\gamma \in \mathfrak{R}^{+}$.

Note from Eq. (3) that the synthetic image results from the $3 \mathrm{D}$ convolution between the object function and the synthetic PSF. This implies that the system is linear and shift-invariant in intensity, so that it can be properly called an imaging system.

Since our aim is the improvement of the axial resolution in the image, it is convenient to analyze the axial component of the synthetic 3D PSF, which is governed by the function

$h_{i}(r=0, z)=\int_{0}^{\alpha} A_{i}(\theta) \exp \left(\mathrm{i} 2 \pi n \frac{\cos \theta}{\lambda_{\text {exc }}} z\right) \sin \theta \mathrm{d} \theta$.

If we perform the non-linear mapping (Martínez-Corral et al., 2002)

$\zeta=\frac{\cos \theta-\cos \alpha}{1-\cos \alpha}-0.5 ; q(\zeta)=A_{i}(\theta)$,

Eq. (5) can be rewritten, neglecting irrelevant constant and phase pre-multiplying factors, as

$h\left(r=0, z_{N}\right)=\int_{-0.5}^{0.5} q(\zeta) \exp \left(\mathrm{i} 2 \pi z_{N} \zeta\right) \mathrm{d} \zeta$,

where the axial position in the focal volume has been expressed in terms of the normalized non-dimensional variable

$z_{N}=\frac{n}{\lambda_{\text {exc }}}(1-\cos \alpha) z$

The non-linear mapping in Eq. (6) can be easily understood in terms of the Ewald sphere $(\mathrm{Gu}, 2000)$ : function $q(\zeta)$ is a linearly scaled version of the axial projection of function $A_{i}(\theta)$. Eq. (7) is an important formula. It indicates that 
the axial amplitude PSF of a lens is given by the 1D Fourier transform of function $q(\zeta)$. This is valid for any value of the semi-angular aperture of the lens, $\alpha$. For a given form of function $q(\zeta)$, the value of $\alpha$ determines both the axial extent of the focal spot (Eq. (8)), and the actual form of the apodization function (Eq. (6)).

\section{The synthetic PSF}

To create an axially superresolving synthetic PSF our proposal relies on the insertion in the collector path 2 of a radially symmetric pupil filter such that its axial PSF has a null at the focus $\left(z_{N}=0\right)$. Then, by subtracting the apodized image from the image obtained with the free pupil, a synthetic image is obtained in which the axial PSF has been importantly narrowed.

To tackle the design of the filter that properly produces zero-focus intensity it is convenient to express function $q(\zeta)$ as a series of Legendre polynomials, $P_{n}(\zeta)$, that are a complete set of orthogonal functions for $-0.5 \leq \zeta \leq 0.5$; that is

$q(\zeta)=\sum_{n=0}^{\infty} a_{n} P_{n}(\zeta)$

Hence by substituting Eq. (9) into Eq. (7) and by taking into account that the 1D Fourier transform of the $n$ order Legendre polynomial is proportional to the spherical Bessel function of the same order, $j_{n}$, we obtain (Abramowitz and Stegun, 1970)

$h\left(r=0, z_{N}\right)=\sum_{n=0}^{\infty}(-\mathrm{i})^{n} a_{n} j_{n}\left(\pi z_{N}\right)$

The apodizer for achieving zero-focal intensity is obtained by recognizing that the first-order spherical Bessel function has the lowest spread among the non-zero-order functions of the same kind. Consequently, the mapped transmittance of our proposed apodizer is in the form $q(\zeta)=P_{1}(\zeta)=2 \zeta$, whose

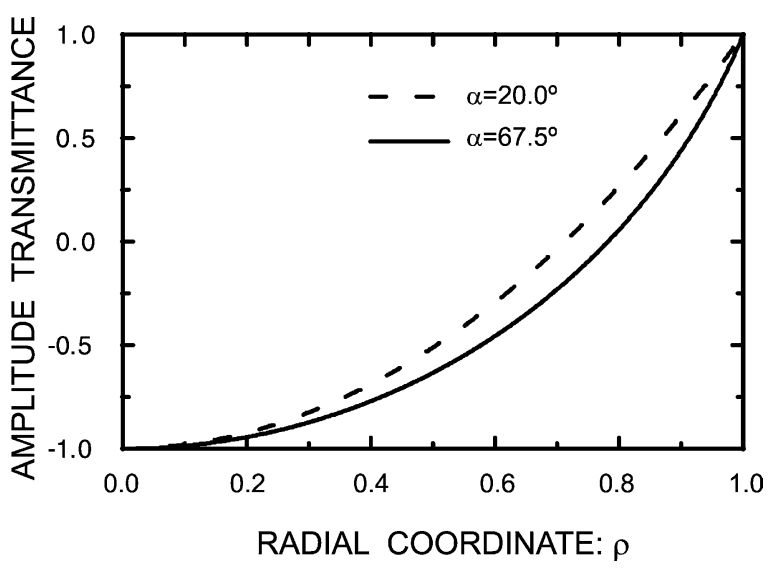

Fig. 2. Amplitude transmittance of the pupil filter that provides zero-focal intensity with the lowest spread. The actual form of the filter depends on the value of the semi-angular aperture of the objective. The radial coordinate is normalized to its maximum actual value. The solid curve corresponds to the case of $\alpha=67.5^{\circ}$, the dashed line to $\alpha=20.0^{\circ}$.

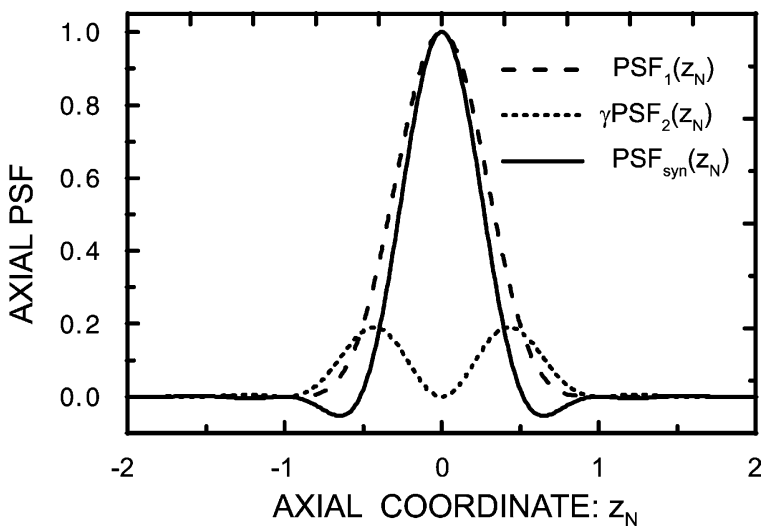

Fig. 3. Numerically evaluated axial intensity PSF corresponding to: onepinhole confocal microscope (dashed line), the path 2 of the twopinhole system (dotted curve); and the synthetic imaging system (solid line). The maximum negative value in the synthetic PSF is -0.05 . The axial positions are specified in terms of the normalized axial coordinate $z_{N}=n(1-\cos \alpha) z / \lambda_{\mathrm{exc}}$. For the calculations we set $\varepsilon=0.9$.

representation with respect to the radial coordinate is shown in Fig. 2.

Next in Fig. 3 we show the PSF-synthesis process. With the dashed line we have represented the function $\operatorname{PSF}_{1}\left(r=0, z_{N}\right)$, which is just the axial PSF of a conventional one-pinhole confocal fluorescence microscope. With the dotted line we have plotted $\gamma \mathrm{PSF}_{2}\left(r=0, z_{N}\right)$. As expected this PSF has a null at the focus. Finally, the solid curve represents the synthetic PSF. After a comprehensive empirical study we determined that $\gamma=1.5$ provides an optimum result. Although our setup was specifically designed to improve the axial resolution, a slight improvement of resolution in the transverse direction is also achieved (Fig. 4).

Finally, in Fig. 5 we compare, in contour color plots, the 3D intensity distribution in a meridian plane corresponding to both the one-pinhole and the two-pinhole confocal setups. The calculations were done according to Eqs. (1)-(3). The figure clearly shows the important compression of the PSF along the axial direction.

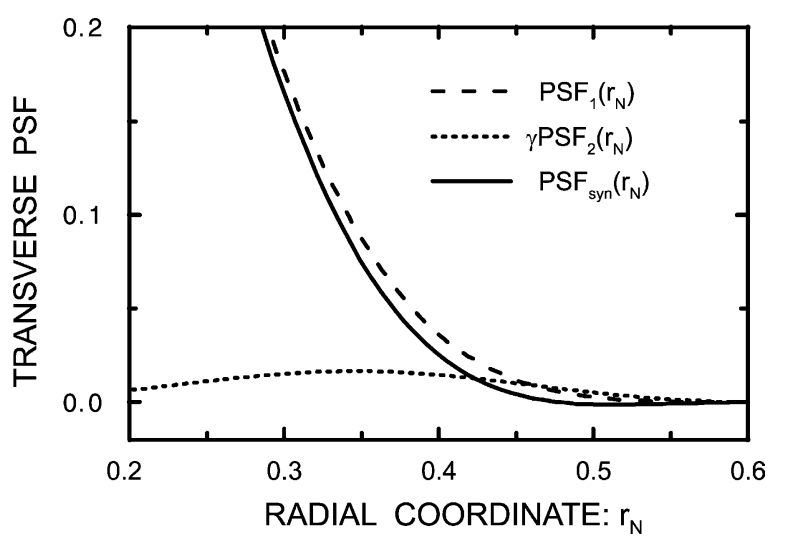

Fig. 4. Transverse PSFs corresponding to the same cases as in Fig. 3. The normalized radial coordinate is $r_{N}=\left(n / \lambda_{\text {exc }}\right) r \sin ^{2} \alpha$. The maximum negative value in the synthetic PSF is -0.001 . 

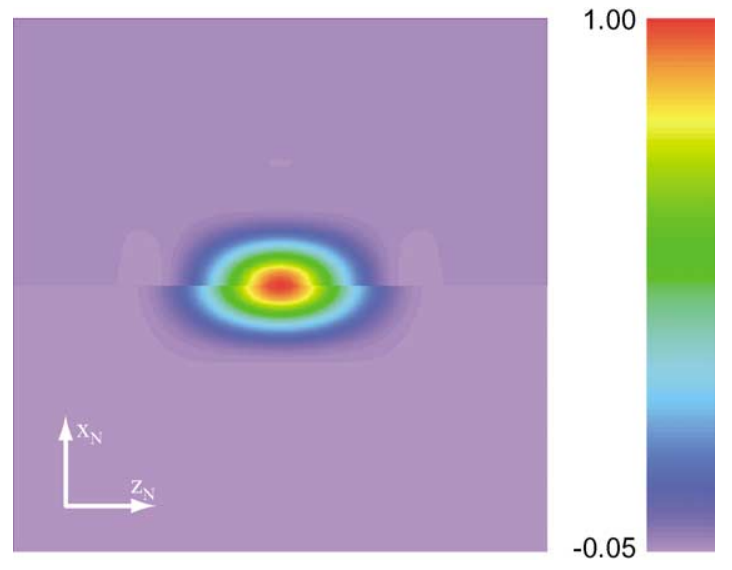

Fig. 5. Numerically evaluated contour plot of the 3D intensity PSF in the meridian plane corresponding to the one-pinhole (bottom) and to the twopinhole (top) confocal microscope.

\section{Table 1}

List of PSF and integrated intensity figures corresponding to conventional one- and two-pinhole confocal scanning microscope

\begin{tabular}{llllll}
\hline & Axial PSF & & & \multicolumn{2}{l}{ Integrated intensity } \\
\cline { 2 - 3 } & $\begin{array}{l}\text { Peak width at } \\
\operatorname{PSF}\left(z_{N}\right)=0.01\end{array}$ & FWHW & & $\begin{array}{l}\text { Peak width at } \\
I_{\text {int }}\left(z_{N}\right)=0.01\end{array}$ & FWHW \\
\hline $\begin{array}{l}\text { One-pinhole } \\
\text { confocal }\end{array}$ & $1.54(100 \%)$ & $0.68(100 \%)$ & $1.78(100 \%)$ & $0.68(100 \%)$ \\
$\begin{array}{l}\text { Two-pinhole } \\
\text { confocal }\end{array}$ & $1.02(66 \%)$ & $0.54(79 \%)$ & $1.34(75 \%)$ & $0.58(85 \%)$ \\
\hline
\end{tabular}

In Table 1 we list and compare the different figures of merit corresponding to two-pinhole and conventional onepinhole confocal fluorescence microscopes.

\section{Numerical imaging experiments}

To illustrate the utility of our proposal we performed two different numerical imaging experiments. First we

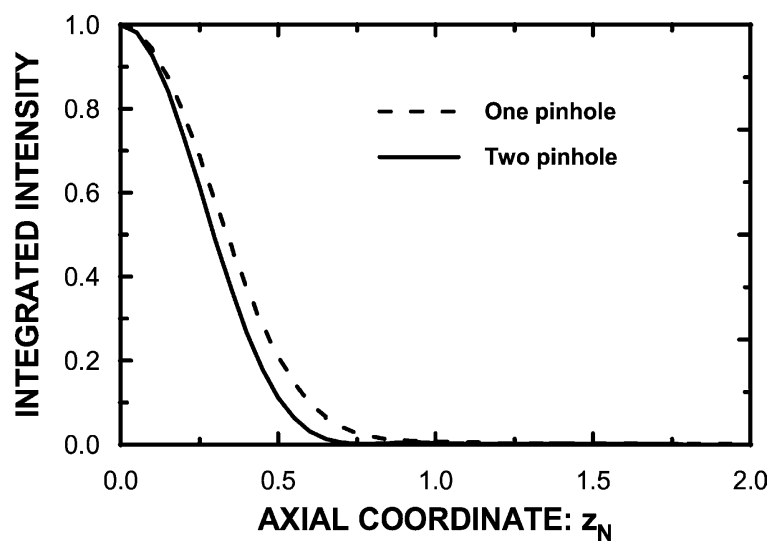

Fig. 6. Integrated intensity for the systems under study. The use of the twopinhole architecture reduces the axial extent of the integrated intensity to a $75 \%$ (measured at $1 \%$ of the maximal intensity).

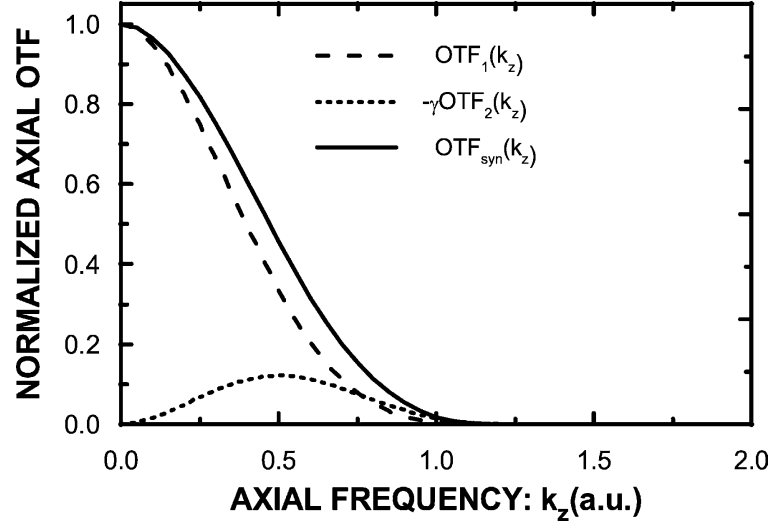

Fig. 7. Axial OTF of the systems under study. Note that the synthetic OTF always takes positive values.

calculated the image of an axially scanned thin fluorescent layer. That is, we calculated the so-called integrated intensity function. This is the key merit function when evaluating the optical sectioning capacity of an imaging system. In Fig. 6 we show the calculated integrated intensity. Note that a $25 \%$ narrowing of the width taken at $1 \%$ of the maximal intensity is achieved.

When dealing with 3D imaging systems, the optical sectioning capacity is usually illustrated by plotting the axial OTF, which results from 1D Fourier transforming the integrated intensity. In Fig. 7 we show how the two-pinhole confocal setup enhances the higher-frequencies contribution.

For our second experiment we investigated the imaging performance of the two-pinhole confocal system by calculating the resulting 3D image of a test object. We designed an elaborated 3D object consisting of two concentric spherical fluorescence labeled shells, as shown in Fig. 8a. The test object was designed to contain all the axial and transverse frequencies of interest. The dark band in the object will permit us to clearly visualize the improvement in resolution along the different directions passing through the focus. After convolution with the confocal PSF and with the two-pinhole synthetic PSF (represented in Fig. 5), we obtained the simulated images shown in Fig. $8 \mathrm{~b}$ and c. Since the synthetic PSF has negative values, in the calculation we suppressed all the negative intensities we got in the image. Note from these figures that a slight improvement in the transverse direction is achieved. Concerning the axial direction, note that the amount of blur in the image of the test-object dark band (devoid of fluorescence) is significantly lower in case of the twopinhole setup.

\section{Conclusions}

An extension of the concept of two-pinhole confocal imaging to the incoherent case has been presented. Specifically what it is new here is: the use of two closed pinholes, and the improvement of resolution in the axial 


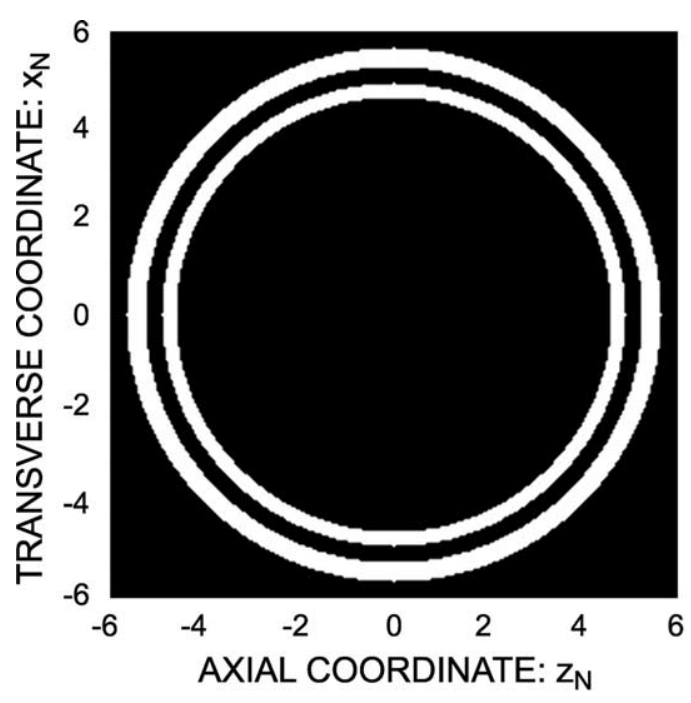

(a)

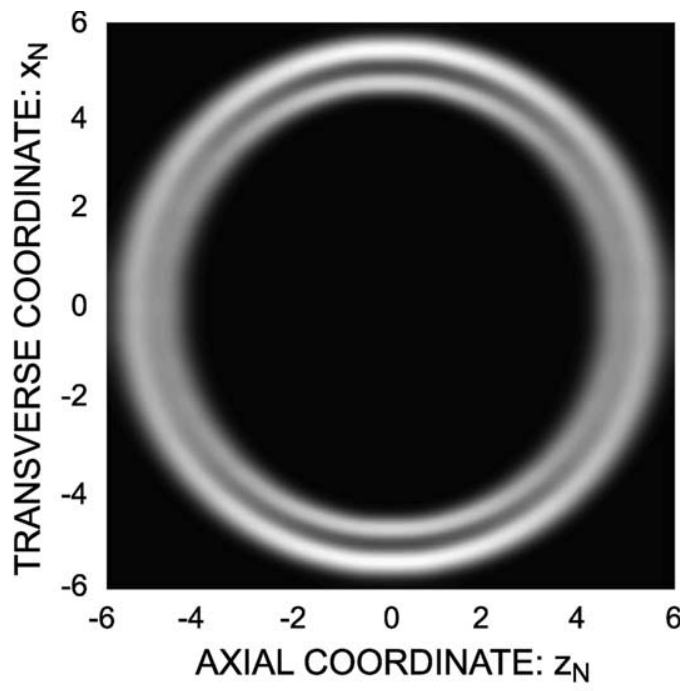

(b)

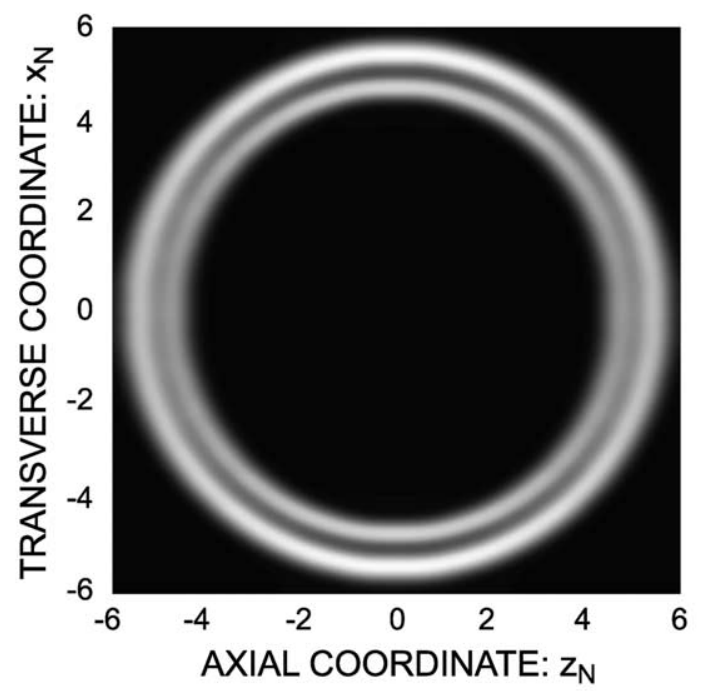

(c)

Fig. 8. Imaging of the elaborated 3D object. (a) the test object consists of two concentric spherical fluorescence labeled shells; (b) Axial section ( $\left.x_{N}, z_{N}\right)$ of the calculated image for the case of the one-pinhole setup; (c) same as (b) but for the case of the two-pinhole confocal setup. Note that both the axial and the transverse distances are expressed in normalized coordinates. The normalized radii for the shells are: inner fluorescence shell, $z_{N}^{\text {in }}=4.68$ and $z_{N}^{\text {out }}=4.98$; outer fluorescence shell, $z_{N}^{\text {in }}=5.25$ and $z_{N}^{\text {out }}=5.65$. The actual dimensions of the numerical experiment depends on both the $\lambda_{\text {exc }}$ and the objective NA.

direction. The cornerstone of our subtractive imaging system is the insertion, in one of the collector paths, of a specially designed zero-focus pupil filter. The amplitude transmittance of the filter depends on the values of both $\alpha$ and $\varepsilon$. The resulting synthetic PSF is substantially narrowed by $34 \%$ (measured at $1 \%$ of the maximal intensity) along the axial direction. If, conversely, we evaluate the optical sectioning capacity in terms of the integrated intensity the narrowing is by $25 \%$ (at $1 \%$ ). The power of our approach was illustrated by a numerical imaging experiment, which showed the superior optical sectioning capacity of the two-pinhole setup. It should be mentioned however, that the suppression of negative values in the final intensity image might lead to sharp images but at the danger of suppressing low intensity features next to bright structures. Therefore, the optimum applicability of the proposed technique should take into account (as it is done in many other superresolving technique) the characteristics of the object.

\section{Acknowledgements}

The authors are indebted to an anonymous reviewer for his/her valuable suggestions, which substantially improved the paper. This work was supported the Plan Nacional I + D + I (Grant DPI2000-0774), Ministerio de Ciencia y Tecnología, Spain. C. Ibáñez-López gratefully acknowledges the financial support from the same institution. 


\section{References}

Abramowitz, M., Stegun, I.A. (Eds.), 1970. Handbook of Mathematical Functions, Dover, New York.

Boyer, G., Sarafis, V., 2001. Two pinhole superresolution using spatial filters. Optik 112, 177-179.

Gu, M., 2000. Advanced optical imaging theory, Springer, Berlin.

Hell, S., Stelzer, E.H.K., 1992. Properties of a 4Pi confocal fluorescence microscope. J. Opt. Soc. Am. A 9, 2159-2166.

Martínez-Corral, M., Kowalczyk, M., Zapata-Rodríguez, C.J., Andrés, P., 1998. Tunable optical sectioning in confocal microscopy by symmetrical defocusing and apodization. Appl. Opt. 37, 6914-6921.
Martínez-Corral, M., Andrés, P., Zapata-Rodríguez, C.J., Kowalczyk, M., 1999. Three-dimensional superresolution by annular binary filters. Opt. Commun. 165, 267-278.

Martínez-Corral, M., Caballero, M.T., Stelzer, E.H.K., Swoger, J., 2002. Tailoring the axial shape of the point spread function using the Toraldo concept. Opt. Express 10, 98-103.

Neil, M.A.A., Juskaitis, R., Wilson, T., Laczik, Z.J., Sarafis, V., 2000. Optimized pupil-plane filters for confocal microscope point-spread function engineering. Opt. Lett. 25, 245-247.

Sheppard, C.J.R., 1999. Binary optics and confocal imaging. Opt. Lett. 24, 505-506.

Wilson, T. (Ed.), 1990. Confocal Microscopy. Academic Press, London. 